IOS Press

\title{
Ethics Review
}

\section{Research Use of Ecological Momentary Assessment for Adverse Event Monitoring Following Amyloid- $\beta$ Results Disclosure}

\author{
Meghan K. Mattos ${ }^{\mathrm{a}, *}$, Susan M. Sereika ${ }^{\mathrm{b}}$, Scott R. Beach ${ }^{\mathrm{c}}$, Hyejin Kim ${ }^{\mathrm{d}}$, William E. Klunk ${ }^{\mathrm{e}}$, \\ Melissa Knox ${ }^{\mathrm{d}}$, Neelesh K. Nadkarni ${ }^{\mathrm{f}}$, Lisa S. Parker ${ }^{\mathrm{g}}$, J. Scott Roberts ${ }^{\mathrm{h}}$, Richard Schulz ${ }^{\mathrm{i}}$, \\ Lisa Tamres $^{\mathrm{d}}$ and Jennifer H. Lingler ${ }^{\mathrm{j}}$ \\ ${ }^{a}$ Acute and Specialty Care, University of Virginia School of Nursing, Charlottesville, VA, USA \\ ${ }^{\mathrm{b}}$ Departments of Health and Community Systems, Epidemiology and Biostatistics, University of Pittsburgh \\ School of Nursing, Graduate School of Public Health and Clinical Translational Science Institute, \\ Pittsburgh, PA, USA \\ ${ }^{\mathrm{c}}$ University Center for Social \& Urban Research, University of Pittsburgh, Pittsburgh, PA, USA \\ ${ }^{\mathrm{d}}$ University of Pittsburgh School of Nursing, Pittsburgh, PA, USA \\ ${ }^{\mathrm{e}}$ Department of Psychiatry, University of Pittsburgh, Pittsburgh, PA, USA \\ ${ }^{\mathrm{f}}$ Department of Medicine, Division of Geriatric Medicine and Gerontology, University of Pittsburgh, \\ Pittsburgh, PA, USA \\ ${ }^{\mathrm{g}}$ Center for Bioethics \& Health Law, University of Pittsburgh, Pittsburgh, PA, USA \\ ${ }^{\mathrm{h}}$ Department of Health Behavior \& Health Education, University of Michigan, School of Public Health, \\ Ann Arbor, MI, USA \\ ${ }^{\mathrm{i}}$ Departments of Psychiatry, Epidemiology, Sociology, Psychology, Community Health, Nursing, \\ Health and Rehabilitation Sciences; University Center for Social and Urban Research; \\ Center for Caregiving Research, Policy and Training; Geriatric Education Center of Pennsylvania; \\ Aging Institute of UPMC Senior Services, University of Pittsburgh, Pittsburgh, PA, USA \\ ${ }^{\mathrm{j}}$ Department of Health and Community Systems, University of Pittsburgh School of Nursing, Pittsburgh, PA, USA
}

Handling Editor: Allyson Rosen

Accepted 11 June 2019

\begin{abstract}
As calls for transparency in human subjects research grow, investigators conducting Alzheimer's disease (AD) biomarker research are increasingly required to consider their ethical obligations regarding the return of AD biomarker test results to research participants. When disclosing these test results to potentially vulnerable participants, investigators may face unique challenges to identify adverse events, particularly psychological events. The purpose of this paper is to describe our research team's experience with developing and implementing a process for enhanced adverse event monitoring following
\end{abstract}

\footnotetext{
*Correspondence to: Meghan K. Mattos, PhD, Assistant Professor, Acute and Specialty Care, University of Virginia School of Nursing, McLeod Hall- Room 4010, PO BOX 800782,
} 
the return of amyloid- $\beta(A \beta)$ imaging results to research participants with mild cognitive impairment (MCI). Ethical and logistical considerations are presented along with preliminary findings from an ongoing randomized controlled trial of $A \beta$ imaging results disclosure in MCI. Following receipt of amyloid imaging results, participants underwent 14 days of adverse event monitoring using ecological momentary assessment (EMA), a strategy to capture health, behaviors, and mood as they occur in participants' natural settings in real time. EMA telephone calls were placed at random during waking hours to screen for mood changes. Investigators were alerted for positive depression, anxiety, suicidal ideation screenings, or for two days of failed call attempts. Preliminary feasibility of twenty-four participants with MCI who participated in EMA mood assessments was successfully completed $83 \%$ ( $\mathrm{SD}=0.4$ ) of the time over 14 days with no alerts for anxiety or depression screening items. EMA, when used with standard adverse event monitoring, is a promising and novel approach to maximize early detection of negative psychological reactions following AD biomarker results disclosed in research settings.

Keywords: Amyloid, cognitive dysfunction, ecological momentary assessment, ethics, research subjects

\section{AMYLOID- $\beta$ IMAGING IN MCI}

Testing for biological markers of Alzheimer's disease $(\mathrm{AD})$ is a nearly ubiquitous practice in clinical research on cognitive aging. As calls for transparency in human subjects research grow, investigators conducting $\mathrm{AD}$ biomarker research are increasingly required to consider their ethical obligations regarding the return of these test results to research participants. In instances where the return of $\mathrm{AD}$ biomarker research results is either ethically required (e.g., when used as the basis for clinical trial enrollment) or otherwise ethically permissible (e.g., valid, significant, and interpretable results are available), the protocol for results disclosure must include sufficient plans for follow-up monitoring of those who receive positive test results (i.e., results with a less favorable prognosis). The purpose of this paper is to describe our research team's experience with developing and implementing a process for enhanced adverse event monitoring following the return of amyloid- $\beta(A \beta)$ imaging results to research participants with mild cognitive impairment (MCI).

$\mathrm{A} \beta$ imaging (AI) is a positron emission tomography (PET) scanning technique that uses radioligands to detect the presence of neuritic plaques associated with $\mathrm{AD}$ in vivo [1]. Although three $\mathrm{A} \beta$ imaging ligands are approved by the U.S. Food and Drug Administration, at present, these agents are primarily used in research rather than in clinical settings. AI results have not traditionally been disclosed to research participants, but there has been a recent interest in returning these results under certain circumstances [2, 3], reflecting a broader trend of increased return of individual research results [4]. As outlined by Lingler and Klunk (2013) [3], sharing these test results with participants who have MCI, in particular, carries unique ethical considerations and requires investigators to address human participants protections with a focus that emphasizes verifying comprehension of the information conveyed and attending to participants' and care partners' psychological reactions.

\section{ADVERSE EVENT MONITORING IN MCI}

Effective adverse event monitoring by research teams is a key safeguard for ensuring that psychological and other risks to participants are minimized (45 CFR 46.111 [OHRP]) by virtue of prompt detection and timely follow up by team members. Nearly all protocols for the disclosure of AI results call for some type of monitoring of participants' psychological outcomes following results disclosure. Such protocols generally require follow-up assessments of mood at standard intervals in the short-term (e.g., 4-6 weeks after disclosure) and for six to twelve months thereafter [5]. In MCI, the potential for recall bias owing to participants' amnestic deficits poses a major threat to internal validity when collecting this critically important safety data. An assessment delay of days or weeks may be especially problematic for capturing fluctuating mood states. This potential issue with data integrity for participants with MCI is compounded by the possibility that receiving $\mathrm{AI}$ results may entail the receipt of potentially life-changing information about risk for progression to the incurable neurodegenerative condition of AD. Proxy reporting by study partners (typically family members of the primary participant) is an alternative to self-report, but assumes that participants fully share their thoughts and feelings with their family members or other care partners. This is not ideal as persons with cognitive impairment may not consistently report changes in mood to their loved ones. Additionally, proxy reporting by study partners may 
overestimate negative patient mood. In a study examining caregiver rating bias, Schulz et al. found that caregivers of early-AD patients reported that patients experienced significantly higher levels of psychological, existential, and physical suffering and lower levels of quality of life than the early-AD patients reported [6]. One approach to overcoming these potential barriers to detecting adverse psychological events following AI results disclosure is to augment traditional adverse event monitoring procedures with ecological momentary assessment (EMA).

\section{ECOLOGICAL MOMETARY ASSESSMENT}

Employing more frequent assessment intervals than traditional assessment approaches allow, EMA is a strategy used in multiple research settings to capture health and other human behaviors, mood fluctuations, and a host of symptoms as they occur in participants' natural settings [7]. Prior to the advent of EMA, participant data about day-to-day experiences were typically collected through paper diaries, behavioral observation, and/or physiological data monitoring at study-determined assessments [8]. However, recent technologic advances make it possible to more accurately capture in-the-moment experiential data in real-time during participants' execution of typical daily routines. EMA includes a variety of electronic diary approaches and technologies to "collect data on a schedule (e.g., daily diaries at a particular time, assessments scheduled at random) or in response to clinical events (e.g., symptom episodes, behaviors)" [7]. In a review of EMA use in aging research [9], three categories of EMA use were identified: 1) as a comparison with global measures, 2) as an outcome measure, and 3) for hypothesis testing of causal models. The most common research use of EMA data collection has been for affect, with compliance rates ranging from 50-90\% [7]. The review also found that cognitive impairment was a common exclusion criterion across studies using EMA. Concerns about capacity to consent and adherence to research protocols may contribute to this exclusion; however, there are ways in which EMA may be modified to facilitate uptake in this population. For example, providing reminders or alerts can improve adherence. An even more effective way to collect such data may be combining the reminder and data collection at one time point, such as a phone ring as the reminder coupled with the call itself to collect data. Examples of this dual approach have been used across fields and disciplines $[10,11]$.

In a review of EMA in mood disorders research [12], most studies analyzed and compared data at the group level, which overlooks the individual-level symptoms or context of the data of interest. One advantage of EMA is having repeated observations in individuals. Rather than having the participant burden of repeated in-office visits, EMA provides the opportunity to report mood or activities in real-time rather than relying on retrospective questionnaires or upon participants to access study materials for self-report. A greater saturation of data also allows for further exploration of within-person mood and symptom variation. EMA can also rigorously monitor potential psychological risks, such as depression $[10,13]$ and provide a viable alternative to assess for fluctuating moods and impaired memory in this at-risk population following disclosure of AI results.

Studies assessing mood or depressive symptoms using EMA are typically descriptive or intervention research studies [11, 13]. However, monitoring for adverse events related to mood in real-time has not been utilized previously for the purpose of adverse event monitoring. An in-depth example of the application of EMA in the novel context of adverse event monitoring for research participants with mild cognitive impairment following AI results disclosure is presented.

\section{EMA FOR RISK REDUCTION}

Use of EMA for adverse event monitoring is part of an ongoing parent study, "Return of Amyloid Imaging Results" (R01AG046906, PI: Lingler). The purpose of this study is to examine the impact of disclosing A $\beta$ PET results to individuals with MCI. The study hypothesized that the value in providing information and choices to potentially vulnerable research participants outweighs the potential harms. The study and methods critical to understanding the context and use of EMA are summarized below.

Study participants with MCI and their care partners (dyad) were recruited in collaboration with a longitudinal cohort study at University of Pittsburgh Alzheimer Disease Research Center (ADRC; P50 AG05133, PI: Lopez). This recruitment strategy assured that all participants had undergone the necessary clinical research interviews and neuropsychological testing to determine MCI diagnosis prior to being recruited for the study. Inclusion criteria 
required that participants were adults with a current ADRC diagnosis of MCI and a willing study partner. Exclusion criteria were having no prior biomarkerbased knowledge of their risk of AD or elevated mood symptoms at baseline (i.e., score $>40$ on the Spielberger State Anxiety Inventory [14], score $\geq 16$ on the Center for Epidemiologic Studies Depression Scale [15], or endorsement of active suicidal ideation using the Beck Scale for Suicidal Ideation [16]).

Following informed consent, dyads were randomized to either the control group (those receiving educational information only) or the intervention/AI group (those receiving educational information and the choice to undergo AI). Dyads from both groups met with a Master's-prepared nurse or counselor to participate in either an "Education Session" (control group), or a "Pre-Test Counseling Session" (intervention group). During the Pre-Test Counseling Session, material intended to aid in the participants' decisionmaking regarding AI was introduced.

If the study participant chose to undergo AI, then a F[18]-florbetapir PET scan was performed. These scans results were independently reviewed and visually rated by three trained readers, who were required to achieve consensus, either positive (i.e., results with a less favorable prognosis) or negative, for significant buildup of $A \beta$ identified. Next, the dyad met with a scan discloser who was randomly selected from a group of three disclosers to present the results.

Results disclosure sessions followed procedures as outlined by Lingler et al. [17]. The disclosers used the same approach to framing positive and negative results, "Your scan showed (or did not show) a significant level of $A \beta$ build up present in your brain." Next, disclosers provided an AD risk estimate based on the presence or absence of $A \beta$ buildup. Limitations of this risk estimate were described, and an EMA follow-up plan was presented. Since follow-up EMA calls would be made to participants over a period of 14 days, staff asked whether the participant would prefer calls on a home or cell phone. If needed, a cell phone was provided by the study during this EMA period.

\section{EMA PROTOCOL}

Follow-up EMA telephone calls were made to monitor adverse events after the disclosure of scan results. The EMA calls were designed to be brief and focused on the period of greatest risk for adverse events. Prior research suggests that adverse psy- chological events are most likely to occur within 10 days of receiving results of predictive testing for neurodegenerative disorders [18]. Comprehensive mood assessments were collected at four points throughout the 12-month study, and EMA telephone interviews supplemented those results. The EMA interviews, with each call typically lasting 5 minutes or less, were conducted for 14 days following receipt of scan results. The purpose of these telephone interviews was to briefly assess, in real time, the participants' current emotional state. EMA mood data were collected approximately once per day. During this follow-up period, participants were called by trained research assistants from the University of Pittsburgh University Center for Social and Urban Research (UCSUR) 12 times over 14 days at random times during waking hours (9:00 am to 9:00 pm), Monday through Saturday. If the participant could not be reached by telephone (i.e., an unsuccessful call attempt), then the call was repeated up to two additional times in the same day. After three unsuccessful call attempts in one day, a message was left on the participant's answering service with a call-back number and call-back instructions. After two days of no contact, the UCSUR staff would notify the research staff to check on the participant's status. An answering service was not an eligibility criterion, and the option to wait for the calls to resume the next day was also an option for participants.

Figure 1 presents the 7-item EMA mood questionnaire, as adapted with permission from the Patient Health Questionnaire-4 (PHQ-4) [19], as administered for the EMA interviews. The PHQ-4 anxiety symptoms were assessed through two subscale items; depressive symptoms were also assessed through two subscale items. In addition, suicidal ideation was assessed through one item; and two "positively worded" items were included in the daily questionnaire to offset the otherwise negatively-worded subscale items. The "positively-worded items" had not been previously validated and were not included in the scoring. Table 1 presents the required actions for EMA administrator staff, depending on the score of the EMA mood assessment. EMA administrator staff entered survey responses into a database programmed to prompt notification of the PI for any flagged responses, as a flagged response may indicate suicidal ideation or severe depression or anxiety. Per the study protocol, any flagged response would require a member of the research team to contact the participant within 24 hours to determine the need for a clinical assessment and individualized follow-up. 


\begin{tabular}{|c|c|c|c|c|}
\hline Over the past day...* & $\begin{array}{l}\text { Not at } \\
\text { all }\end{array}$ & $\begin{array}{c}\text { A little } \\
\text { of the } \\
\text { time }\end{array}$ & $\begin{array}{c}\text { A lot } \\
\text { of the } \\
\text { time }\end{array}$ & $\begin{array}{c}\text { Nearly } \\
\text { all } \\
\text { of the } \\
\text { time }\end{array}$ \\
\hline 1. I enjoy my friends ${ }^{\mathrm{a}}$ & O & O & O & O \\
\hline 2. I have been feeling nervous anxious or on edge $\mathrm{e}^{\mathrm{b}}$ & O & O & O & O \\
\hline 3. I have not been able to stop or control worrying ${ }^{b}$ & O & O & O & O \\
\hline $\begin{array}{l}\text { Overall I expect more good things to happen to me than } \\
\text { bad }^{\text {a }}\end{array}$ & $\mathrm{O}$ & O & O & O \\
\hline 5. I have been feeling down, depressed, or hopeless ${ }^{\mathrm{c}}$ & $\mathrm{O}$ & O & O & O \\
\hline 6. I have had little interest or pleasure in doing things ${ }^{\mathrm{c}}$ & O & O & O & O \\
\hline 7. The thought of harming myself has occurred to $m e^{d}$ & & yes & no & \\
\hline \multicolumn{5}{|c|}{ 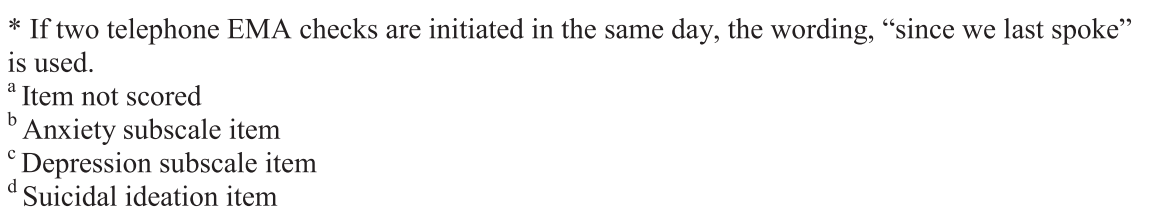 } \\
\hline
\end{tabular}

Fig. 1. Ecological momentary mood assessment questionnaire items.

Table 1

EMA alert follow-up actions for staff

\begin{tabular}{|c|c|c|}
\hline EMA response/ alert & UCSUR staff action & Research team member action \\
\hline $\begin{array}{l}\text { Score of } 4 \text { or more on either subscale for } \\
3 \text { consecutive days. (Anxiety subscale: } \\
\text { items } 2 \& 3 \text { ) (Depression subscale: } \\
\text { items } 5 \& 6 \text { ) }\end{array}$ & $\begin{array}{l}\text { Contact within one business day (during } \\
\text { business hours): } \\
\text { a. Study main office or, if not available: } \\
\text { b. Study project office or, if not available: }\end{array}$ & $\begin{array}{l}\text { Contact the participant directly within } 24 \\
\text { hours to determine if further action is } \\
\text { necessary. }\end{array}$ \\
\hline $\begin{array}{l}\text { Two consecutive days of having made no } \\
\text { verbal contact with the participant }\end{array}$ & c. Study staff mobile number & \\
\hline A response of yes on item 7 & $\begin{array}{l}\text { Notify the Principal Investigator (PI) } \\
\text { a. If the PI is out of town, her voicemail } \\
\text { will specify the name and number of a } \\
\text { co-I designee. }\end{array}$ & $\begin{array}{l}\text { If deemed necessary, a } \\
\text { clinically-licensed research team } \\
\text { member will follow up to perform an } \\
\text { individualized clinical assessment. }\end{array}$ \\
\hline
\end{tabular}

Data were analyzed using SAS 9.4 (SAS Institute, Inc., Cary, NC). Categorical variables were described using frequencies and percentages; means and standard deviations (SD) were calculated for continuous variables. Trends over time were displayed in time plots for individual participants and summarized by $\mathrm{A} \beta$ results status (positive, negative). Random coefficient modeling was used to estimate and compare temporal trends in depressive and anxiety symptom scores between participants who had a positive $A \beta$ result (i.e., results with a less favorable prognosis) and those with a negative result. The variability in anxiety and depression symptoms scores from call to call within a participant was also computed and compared between participants who had a positive
$\mathrm{A} \beta$ result and those with a negative result using the independent samples $t$-test.

\section{FEASIBILITY OF IMPLEMENTATION OF EMA TELEPHONE INTERVIEWS}

Twenty-four participants with MCI who participated in the ecological momentary mood assessments were frequency matched according to $\mathrm{A} \beta$ status (12 positive/12 negative), race, and age. Participants were on average $75.4(\mathrm{SD}=5.5)$ years of age, had an average of $17.4(\mathrm{SD}=1.8)$ years of education, and had Mini-Mental State Examination scores ranging from 22 to $29($ Mean $=26.63, \mathrm{SD}=1.8)$. Most were 
white $(n=22,91.7 \%)$, half were male $(n=12,50 \%)$, and either retired or not working full-time $(n=20$, $83.3 \%)$.

To assess for preliminary feasibility, we examined the ability of EMA to capture mood in real-time, which included successful data collection and factors contributing to the success or failure to capture the mood via telephone interviews. Preliminary feasibility showed that of the 12 daily contact points for each person during the 14-day EMA period, data collection was successfully completed $83 \%(\mathrm{SD}=14)$ of the time. This required 432 outgoing calls for 240 completed calls representing a 55\% contact rate. To determine resource implications, we used Computer Assisted Telephone Interviewing (CATI) to examine the 432 outgoing calls lasting about 5 minutes. We found that about 36 hours were spent calling and collecting data for this 24-participant sample, which is an average of 1.5 hours of person-hours per participant.

Regarding preferred phone communication, we found that twelve (50\%) participants provided their home phone number as first preference for calls, eleven (46\%) preferred their mobile phone for EMA contact, and one (4\%) elected to use the studyprovided cellular phone. There was no difference in call success between participant contacted via home or cellular phone $\left(\mathrm{Mean}_{\text {(home) }}=86 \%\right.$ versus $\operatorname{Mean}_{(\text {cell })}=81 \%, t=0.962, p=0.346$ ).

Regarding the results of the mood assessments, for all calls to participants, there were seven total "no contact" alerts received for six participants (25\% of participants). In each instance of a "no contact" alert from staff, a research staff member reached the participant and verified safety with 24 hours. No alerts were received for anxiety or depression screening items.

\section{EMA MOOD RATINGS}

Visual inspection of response trajectories for anxiety and depression symptom ratings in the $A \beta$ positive group showed, on average, more variability and a delayed worsening in mood symptoms. Closer inspection of participants' individual trajectory profiles by $A \beta$ status highlights the delayed worsening of mood symptoms for participants when compared to participants with negative results, from the time of result disclosure to the end of the 14-day EMA monitoring period. Through application of random coefficient modeling to examine differences in the mean response trajectories from call to call between patients with a positive result versus patients with a negative result, no statistically significant $(p \geq 0.05)$ main effects for calls (as a marker of time), $A \beta$ status, or call by $\mathrm{A} \beta$ status interactions for either anxiety (calls: $\mathrm{F}=0.06, p=0.810 ; \mathrm{A} \beta$ status: $\mathrm{F}=1.24$, $p=0.279$; call by $\mathrm{A} \beta$ status interactions: $\mathrm{F}=0.05$, $p=0.817$ ) or depressive symptoms (calls: $\mathrm{F}=0.48$, $p=0.490 ; \mathrm{A} \beta$ status: $\mathrm{F}=0.03, p=0.872$; call by $\mathrm{A} \beta$ status interaction: $\mathrm{F}=0.76, p=0.385$ ) were found. However, examination of the variability in mood ratings from call-to-call within a participant revealed that patients with a positive scan result had significantly more variability in their anxiety symptom ratings over the EMA monitoring period than patients whose scan was negative $\left(\operatorname{Mean}_{(-)}=0.259\right.$ versus $\operatorname{Mean}_{(+)}=0.569, t=-2.103, p=0.047$; see Fig. 2). A trend was also found for patients with $A \beta$ positive results, with greater variability in depressive symptom ratings from call to call compared to patients having a negative scan result $\left(\mathrm{Mean}_{(-)}=0.227\right.$ versus Mean $_{(+)}=0.547, t=-2.021, p=0.056$; see Fig. 3).

\section{EMA TO IDENTIFY POTENTIAL PYSCHOLOGICAL HARM}

These findings suggest that supplementing standard approaches to adverse event monitoring in research settings with the application of EMA to assess mood is both feasible and effective. Assessing mood in real-time using a brief telephone questionnaire may help reduce recall bias and capture fluctuating mood states in psychologically at-risk persons with MCI following disclosure of potentially life-changing AI results. The results demonstrate that EMA affords an opportunity to identify at-risk participants in real-time and to intervene earlier for psychological concerns. This may provide critical psychological state information for both participants and researchers in this burgeoning field of $\mathrm{AI}$ and other AD biomarker results disclosure.

This study documents that older adults with MCI consistently answered their phones and successfully participated in short, 5-minute assessments over the two-week period following results disclosure in this research setting. Previously, concerns have been raised about the use of technology in older adults that might diminish call success [20]; however, this was not observed in the study. Rather, we found that in this older sample, participants with MCI were able to answer random calls throughout the day or a followup call at night. In younger adults with MCI, who may be working full-time, the call success rate may 


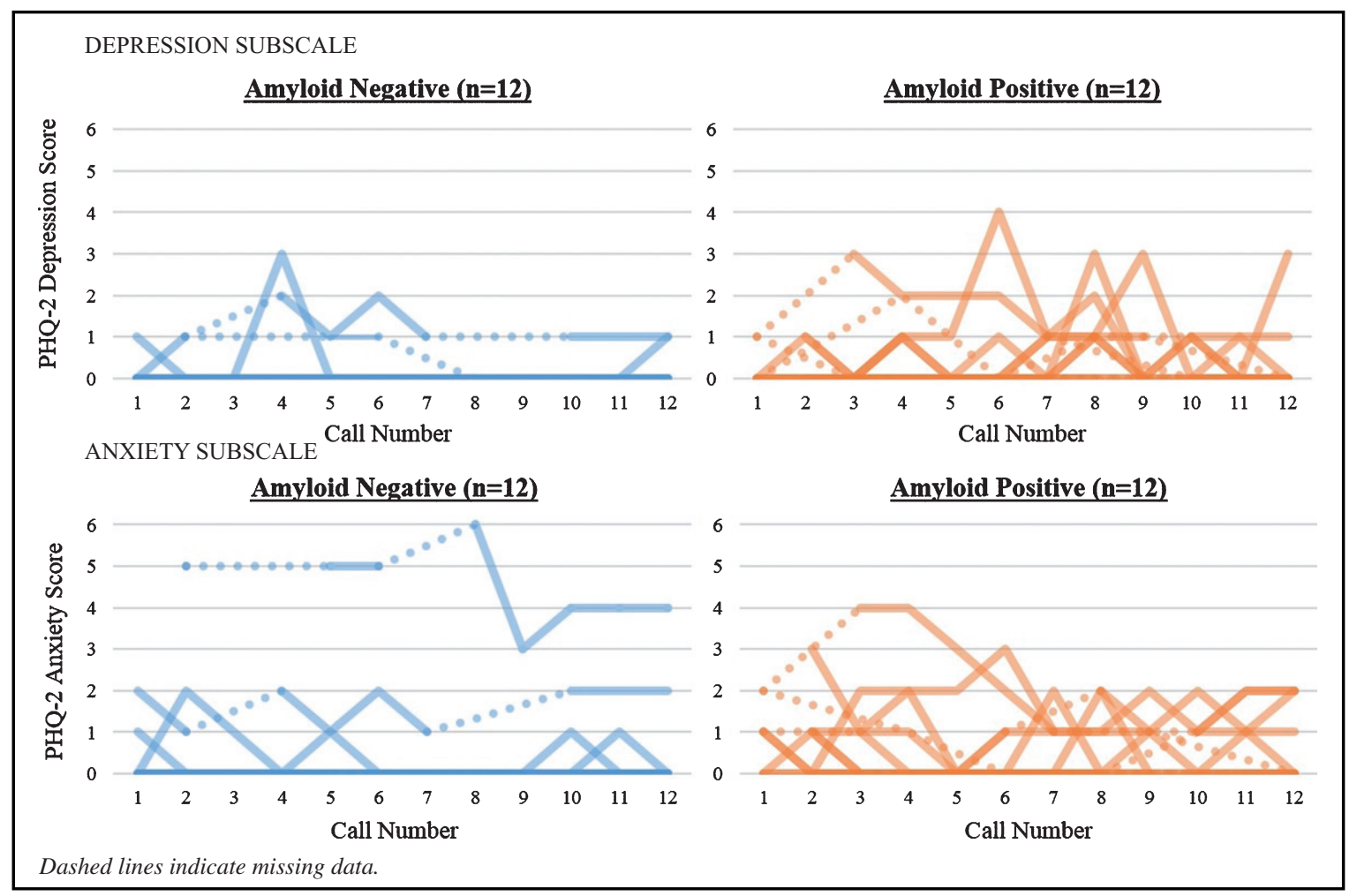

Fig. 2. Individual patient health questionnaire- 4 response profiles by amyloid- $\beta$ status $(N=24)$.

be significantly lower. For follow-up calls, participants could use their own personal cellular phones or could receive a study-provided cellular phone, but in our study, only one participant opted to use the study cell phone. EMA typically collects data on cellular phones while individuals engage in their typical everyday activities. It is conceivable that participants may have spent more time at home anticipating the calls, leading to high rates of call completion. Moreover, this study enrolled an older sample who may have more leisure time than their younger counterparts. If so, then younger persons may have lower call success.

For this study, EMA contact was initiated by a person and required a verbal dialogue between two people. This approach represents a departure from the more common practice in EMA research which relies upon one-way communication with participants and ascertains self-reports via cellular phone applications, e-mail correspondence, or remote/noninvasive sensing. We found that, while admittedly more resource intensive than electronic data capture, relatively few person hours per participant were spent calling and collecting data in our study, and brief,

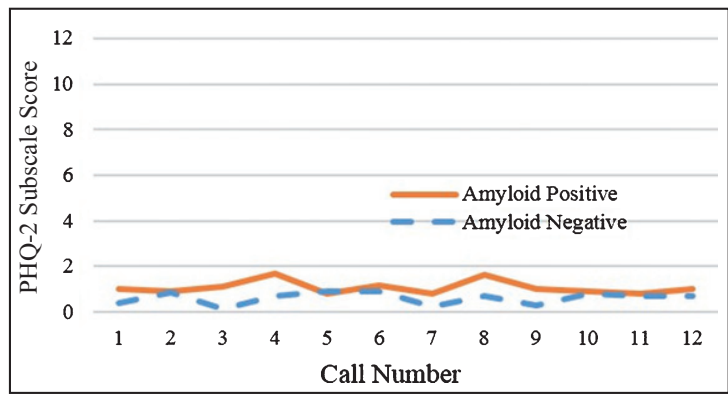

Fig. 3. Mean PHQ-2 depressive symptoms for the total sample $(\mathrm{N}=24)$.

real-time interactions with another person is a highly feasible approach to help identify adverse events in this already vulnerable population. Although about half of the sample requested contact via a cellular phone, we do not know the extent to which cellular phone use extends outside of phone calls to other applications such as email or texting.

It is possible that the use of EMA, as a data collection approach, had an impact on mood. When receiving frequent reminders and study contacts, the use of EMA may dissuade participants from truly 
disclosing their mood or real-time responses due to question conditioning. The frequent questions about mood may promote negative recall of events or fail to capture true representative of a depressive mood due to a variation from baseline [12]. One way to minimize this concern is to consider depression status at baseline, before an intervention is initiated, and over the course of the study.

Preservation of participant privacy is of the utmost importance in human subjects research, especially in individuals with stigmatized conditions such as MCI. Thus, there may be apprehension to use EMA to collect mood data, particularly when contacts/assessments are randomly timed and collected when participants may be surrounded by friends, family, or in public spaces when they receive a notification. For example, a participant may need to carry a cell phone to receive reminders to answer a few questions while at lunch with friends. Depending on the study sample, this may cause discomfort for the participant, such as being asked about the purpose of the alert or who was calling. To minimize this privacy concern, one may ask the participant to identify: 1) the best number to reach them, 2) times to call/not to call, and 3) whether a message can be left at the provided number.

Another consideration when considering return of AI results is a patient's interest in receiving them. For this study, all participants randomized to the imaging group were presented with the opportunity to have $A \beta$ imaging, but participated in pre-test counseling and discussions before deciding whether to undergo a scan. These pre-test counseling discussions focused on promoting informed decision-making related to the $\mathrm{AI}$ and encouraged participants to consider how they might react to various result disclosure scenarios and whether they might make any changes (e.g., lifestyle, advance planning, etc.) based on their results. As more longitudinal studies yield information about the implications of AI results in terms of AD-conversion risk, protocols for promoting informed decision-making around results disclosure may take on even greater significance.

Our findings raise important questions regarding whether the use of EMA for adverse event monitoring may be appropriate in other settings, such as the disclosure of genetic test results or other biomarker testing in clinical practice settings. The intention for use of EMA in our study was to identify mood changes early in the days following the receipt of potentially life-changing imaging results. Before considering use outside of an AI research dis- closure protocol, the use of EMA in non-research settings would require validating EMA risk criteria against established standards for risk. Ultimately, use of EMA for mood assessments would require recommendations for action in response to high risk and an assessment that those actions made a difference in patient outcomes. Before adopting this relatively resource-intensive practice in clinical settings, the approach requires further investigation of its value in relation to alternative methods of monitoring psychological well-being.

\section{FINAL THOUGHTS}

Our experience suggests that it is important to consider the use of EMA in this population of adults with MCI as a way to engage and capture real-time activities and mood to provide safeguards in research. In addition to this novel use of EMA, the inclusion of inperson, clinician-led pre-test counseling and results disclosure sessions provided essential informational and support resources for dyads. These sessions provide the opportunity to field questions, clarification, and promote discussion about undergoing AI and understanding AI results.

EMA mood assessments are a novel way to promote the protection of human participants learning potentially life-changing AD biomarker test findings. EMA, when used with standard adverse event monitoring, is a promising approach to maximize early detection of negative psychological reactions following $\mathrm{AD}$ biomarker results disclosed in research settings. EMA as a standard assessment tool may provide an opportunity to collect important study data, while also monitoring for adverse effects and potential harm for participants.

\section{ACKNOWLEDGMENTS}

Research reported in this publication was supported by National Institute on Aging of the National Institutes of Health under award numbers R01AG046906 (PI: J.H. Lingler) \& P50AG005133 (PI: O. Lopez); Aging Institute of UPMC; and Avid Radiopharmaceuticals, a wholly owned subsidiary of Eli Lilly and Company.

Avid Radiopharmaceuticals supplied Florbetapir F 18 injections for this study at no cost to the research study.

Authors' disclosures available online (https:// www.j-alz.com/manuscript-disclosures/19-0091r2). 


\section{REFERENCES}

[1] Rabinovici GD, Jagust WJ (2009) Amyloid imaging in aging and dementia: Testing the amyloid hypothesis in vivo. Behav Neurol 21, 117-128.

[2] Grill JD, Johnson DK, Burns JM (2013) NIH Public Access. Neurodegener Dis Manag 3, 43-51.

[3] Lingler JH, Klunk WE (2013) Disclosure of amyloid imaging results to research participants: Has the time come? Alzheimers Dement 9, 741-744.

[4] The National Academies of Science Engineering Medicine (2018) Return of Individual-Specific Research Results Generated in Research Laboratories. http://nationalacademies.org/hmd/Activities/Research/Rese archResultsGeneratedinResearchLaboratories.aspx

[5] Burns JM, Johnson DK, Liebmann EP, Bothwell RJ, Morris JK, Vidoni ED (2017) Safety of disclosing amyloid status in cognitively normal older adults. Alzheimers Dement 13, 1024-1030.

[6] Schulz R, Cook TB, Beach SR, Lingler JH, Martire LM, Monin JK, Czaja SJ (2013) Magnitude and causes of bias among family caregivers rating Alzheimer's disease patients. Am J Geriatr Psychiatry 21, 14-25.

[7] Shiffman S, Stone AA, Hufford MR (2008) Ecological momentary assessment. Annu Rev Clin Psychol 4, 1-32.

[8] Stone A, Shiffman S (1994) Ecological momentary assessment (EMA) in behavioral medicine. Ann Behav Med 16, 199-202.

[9] Cain A, Depp C, Jeste D (2009) Ecological momentary assessment in aging research: A critical review. J Psychiatry Res 43, 987-996.

[10] Moore RC, Depp CA, Loebach J, Lenze EJ (2016) Ecological momentary assessment versus standard assessment instruments for measuring mindfulness, depressed mood, and anxiety among older adults. J Psychiatr Res 75, 116123.
[11] Wenze SJ, Miller IW (2010) Use of ecological momentary assessment in mood disorders research. Clin Psychol Rev 30, 794-804.

[12] Aan het Rot M, Hogenelst K, Schoevers RA (2012) Mood disorders in everyday life: A systematic review of experience sampling and ecological momentary assessment studies. Clin Psychol Rev 32, 510-523.

[13] Armey MF, Schatten HT, Haradhvala N, Miller IW (2015) Ecological momentary assessment (EMA) of depressionrelated phenomena. Curr Opin Psychol 4, 21-25.

[14] Spielberger C, Gorsuch R, Lushene R, Vagg P, Jacobs G (1983) Manual for the State-Trait Anxiety Inventory, Consulting Psychologists Press, Palo Alto, CA.

[15] Radloff L (1977) The CES-D scale: A self-report depression scale for research in the general population. Appl Psychol Meas 1, 385-401.

[16] Beck A, Steer R (1991) BSS: Beck Scale for Suicide Ideation, Psychological Corporation.

[17] Lingler JH, Butters MA, Gentry AL, Hu L, Hunsaker AE, Klunk WE, Mattos MK, Parker LA, Roberts JS, Schulz R (2016) Development of a standardized approach to disclosing amyloid imaging research results in mild cognitive impairment. J Alzheimers Dis 52, 17-24.

[18] Lawson K, Wiggins S, Green T, Et A (1996) Adverse psychological events occurring in the first year after predictive testing for Huntington's disease. J Med Genet 33, 856-862.

[19] Kroenke K, Spitzer RL, Williams JBW, Löwe B (2009) An ultra-brief screening scale for anxiety and depression: The PHQ-4. Psychosomatics 50, 613-621.

[20] Heinz M, Martin P, Margrett JA, Yearns M, Franke W, Yang H-I, Wong J, Chang CK (2013) Perceptions of technology among older adults. J Gerontol Nurs 39, 42-51.

[21] Wendler D, Emanuel E (2003) The debate over research on stored biological samples. Arch Intern Med 162, 1457. 